\title{
A Nonlinear Pulsation Model for V652 Her
}

\author{
Yu. A. Fadeyev \\ Institute for Astronomy of the Russian Academy of Sciences, \\ Pyatnitskaya Str. 48, 109017 Moscow, Russia.
}
A. E. Lynas-Gray
Department of Physics, University of Oxford, OX1 3RH, England.

\begin{abstract}
When compared with observation, stellar pulsation model predictions provide a test of stellar envelope opacities. Of specific interest, is the radially pulsating extreme helium star V652 Her for which luminosity and radial velocity observations are published. Nonlinear pulsation models of V652 Her, calculated using Opacity Project (OP) opacities, predict luminosity and radial velocity variations in close agreement with observation.
\end{abstract}

\section{Introduction}

Using radial stellar pulsation theory, linear and nonlinear calculations show V652 Her to be beyond the blue boundary of the extreme helium star pulsation instability region when envelope opacities from the Los Alamos Opacity Library (LAOL, Hübner et al. 1977) are adopted (Saio \& Jeffery 1988, Fadeyev 1993). New stellar envelope opacities (OPAL, Iglesias et al. 1992) enable Saio (1993), using a linear pulsation model, to show the opacity bump at $T \sim 2 \times 10^{5} \mathrm{~K}$ (completely absent from LAOL opacities) to be responsible for driving radial pulsations in V652 Her. OP opacities (Seaton et al. 1994), while in agreement with OPAL, also include significant contributions due to ions of chromium, manganese and nickel; this motivates the present calculation of a nonlinear radial pulsation model for V652 Her with the view to making a comparison with observed radial velocity and luminosity curves.

\section{Nonlinear pulsation model for V652 Her}

Nonlinear radial pulsation models for V652 Her were calculated using Fadeyev's (1983) code and OP opacities computed for mixtures suggested by photospheric abundances obtained by Jeffery et al. (1986). Figure 1 illustrates the best agreement with observation obtained for a $0.72 \mathrm{M}_{\odot}$ star having $\mathrm{T}_{\text {eff }}=23500 \mathrm{~K}$ and composition by numbers of $n(H)=0.006, n(H e)=0.99, n(N)=0.002$ and $n(Z *)=0.002 ; Z *$ represents all metals (except nitrogen) with abundances in proportions given by Anders \& Grevesse (1989). In transforming theoretical 

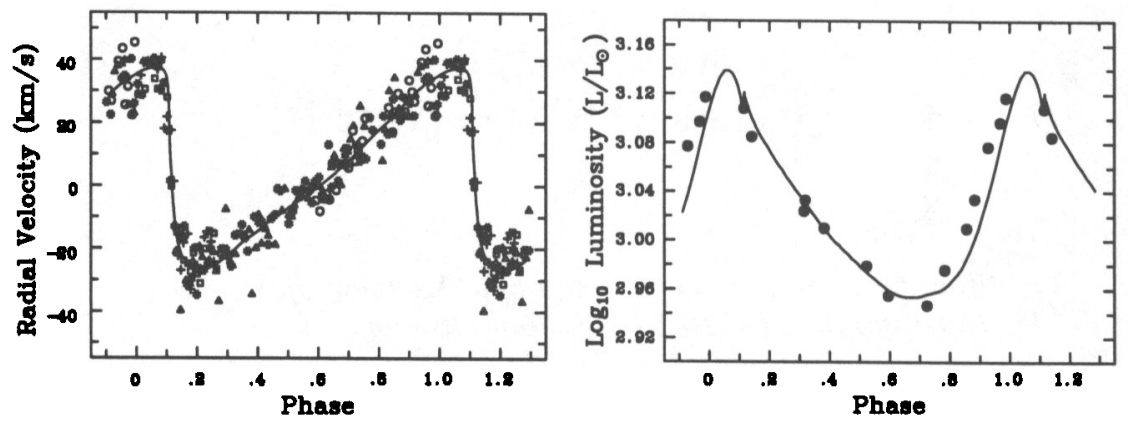

Figure 1. A comparison between observed (points) and calculated (solid line) pulsation variations in V652 Her; different symbols distinguish pulsation cycles. Radial velocity data are from Jeffery \& Hill (1986); luminosities are from Lynas-Gray et al. (1984).

radial velocities from the centre-of-mass frame to the observer's rest frame, a uniform disk (no limb darkening) has been assumed.

\section{Conclusion}

In the case of V652 Her, nonlinear pulsation model calculations are limited by uncertain photospheric abundances and $T_{\text {eff }}$. Nonetheless, the agreement between observation and theory in Figure 1 is remarkably good and verifies the quality of OP opacities.

Acknowledgments. The Austrian Ministry of Science generously awarded a. research grant to fund the HP9000/425e used for nonlinear pulsation model calculations.

\section{References}

Anders, E., \& Grevesse, N. 1989, Geochim. Cosmochim. Acta, 53, 197

Fadeyev, Yu. A. 1983, Nauchn. Inform. Astron. Council, 52, 3

Fadeyev, Yu. A. 1993, MNRAS, 262, 119

Hübner, W.F., Merts, A.L., Magee, N.H., \& Argo, M.F. 1977, Los Alamos Report LA-6760-M

Iglesias, C.A., Rogers, F.J., \& Wilson, B.G. 1992, ApJ, 397, 717

Jeffery, C.S., Heber, U., \& Hill, P.W. 1986, IAU Coll, 87, 101

Jeffery, C.S., \& Hill, P.W. 1986, MNRAS, 221, 975

Lynas-Gray, A.E., Schönberner, D., Hill, P.W., \& Heber, U. 1984, MNRAS, 209, 387

Saio, H. 1993, MNRAS, 260, 465

Saio, H., \& Jeffery, C. S. 1988, ApJ, 328, 714

Seaton, M.J., Yu Yan, Mihalas, D. \& Pradhan, A.K. 1994, MNRAS, 266, 805 